\title{
HACIA UNA OBJETIVIDAD CIENTÍFICA ABIERTA: UNA REFLEXIÓN DESDE LA CONCEPCIÓN EPISTEMOLÓGICA DE EVANDRO AGAZZI
}

\section{TOWARDS AN OPEN SCIENTIFIC OBJECTIVITY: A REFLECTION FROM THE EPISTEMOLOGICAL CONCEPTION OF EVANDRO AGAZZI}

\author{
Robert Fernando Bolaños VIVAS \\ Universidad Politécnica Salesiana del Ecuador \\ Johman CARVAJAL GODOY \\ Universidad Pontificia Bolivariana de Medellin ${ }^{* *}$
}

\begin{abstract}
Resumen: Ante las distintas maneras de entender la objetividad científica, con una clara prevalencia del sentido empírico-experimental, que conlleva cierta discriminación epistémica para con aquellas áreas del conocimiento, cuyos criterios de validez cognitiva son diferentes a los de la contrastación experimental, el profesor Evandro Agazzi, con su realismo científico y su concepción epistemológica abierta e incluyente, configura un concepto de objetividad adecuado para todo quehacer científico y académico. En efecto, con su distinción entre objetividad en sentido débil y fuerte, Agazzi sostiene que estas formas de entender la objetividad pueden incluir y armonizar los variados horizontes epistemológicos desde los cuales es posible hacer ciencia y academia de manera más pertinente y adecuada al contexto contemporáneo, caracterizado por el dinamismo y la complejidad.
\end{abstract}

\footnotetext{
* Docente en la Universidad Politécnica Salesiana del Ecuador; miembro del Grupo de Investigación de Filosofía de la Educación (GIFE); código postal: 170502, rbolanosv@ups.edu.ec, robertfer77@yahoo.es.

** Docente en la Universidad Pontificia Bolivariana, Medellín, Colombia, johman.carvajal@ upb.edu.co.
} 
Palabras clave: Objetividad científica, epistemología, ciencia, intersubjetividad científica, conocimiento.

Aвsтract: Faced with the different ways of understanding scientific objectivity, with a clear prevalence of the empirical-experimental sense, which entails some epistemic discrimination with those areas of knowledge, whose criteria of cognitive validity are different from experimental contrast, Professor Evandro Agazzi, with his scientific realism and open and inclusive epistemological conception, sets up a concept of objectivity suitable for all scientific and academic work. Indeed, with its distinction between objectivity in a weak and strong sense, Agazzi argues that these ways of understanding objectivity can include and harmonize the varied epistemological horizons from which it is possible to do science and academia more relevant and appropriate to the contemporary context, characterized by dynamism and complexity.

KEYwORDs: Scientific objectivity, epistemology, science, scientific intersubjectivity, knowledge.

\section{Introducción}

El presente artículo profundiza sobre la noción de objetividad científica, a la luz del filósofo de la ciencia italiano Evandro Agazzi. ¿Qué se entiende cuando se afirma que un conocimiento es objetivo?, ¿es lo mismo objetividad que comprobación experimental?, ¿̇todas las ciencias pueden alcanzar objetividad?, ¿hay diversos tipos de objetividad científica? La reflexión filosófica en torno a estas preguntas toca los desafíos y los contenidos de la epistemología y de la filosofía de la ciencia contemporánea, en el esfuerzo por la configuración de una ciencia pertinente que preserve y asegure el hábitat en el que desenvuelve la vida y posibilite una vida humana caracterizada por el bienestar.

El problema que se constata es que el predominio pragmático y utilitario de las ciencias experimentales sobre las ciencias humanísticas, hace que, en la actualidad, el sentido más corriente y aceptado de objetividad sea el de comprobabilidad experimental, a través de la cual un conocimiento se considera objetivo cuando se somete al control experimental y termina ofreciendo resultados prácticos y útiles para la colectividad. Ante esta realidad, las ciencias humanísticas, cuya naturaleza no siempre requiere del control experimental, se conciben como saberes de segundo orden, cuyas elucubraciones subjetivas no producen artefactos útiles para la cotidianidad de las personas. Así pues, 
se pone en duda la pertinencia y permanencia de las ciencias humanas en el quehacer científico contemporáneo por su falta de utilidad, e incluso, su rigurosidad y validez argumentativa.

Una pista de solución al problema la ofrece Evandro Agazzi, cuando postula la necesidad de contextualizar la comprensión y aplicación de la objetividad. Esto implica que, en la permanente búsqueda de objetividad, cualquier científico o investigador, debe considerar la naturaleza de cada ciencia y, sobre todo, tener la suficiente preparación epistemológica para que las construcciones científicas que utiliza correspondan a la naturaleza epistemológica de la ciencia en la que desarrolla su actividad.

Una de las limitaciones del presente artículo tiene que ver con el hecho de que Evandro Agazzi presupone que sus interlocutores manejan las nociones y problemáticas de la historia de la ciencia y, por lo tanto, poseen un amplio bagaje de esos conceptos y problemas epistemológicos surgidos en las diversas épocas de la ciencia. En efecto, existe la conciencia de que, sin un manejo suficiente de las nociones y problemas de la ciencia moderna y contemporánea, las reflexiones y posibles soluciones que se ofrecen corren el riesgo de carecer de sentido.

A pesar de esta importante limitación, el objetivo del presente artículo es otorgar elementos para una comprensión más amplia y contextualizada del concepto de objetividad científica para los agentes y operadores de la ciencia. Todo esto para evitar que las ciencias invadan terrenos ajenos a su naturaleza epistemológica específica, ya sea desde lo metodológico, lo procedimental o lo teleológico, en el esfuerzo por configurar un concepto de objetividad científica que incluya y justifique el quehacer investigativo y académico de los diferentes tipos de prácticas científicas contemporáneas.

Los métodos que se siguen son el hermenéutico y el bibliográfico. Se ha realizado un rastreo de los principales escritos de Evandro Agazzi en las que se reflexiona sobre la objetividad científica en su relación con la epistemología contemporánea, con el consiguiente esfuerzo por hacer una interpretación contextualizada de sus significados y sus problemas. 


\section{La objetividad científica en contexto}

El concepto de objetividad se enmarca dentro de las concepciones epistemológicas del realismo científico defendido, entre otros, por Evandro Agazzi (Agazzi, 2009: 46) y se constituye en uno de los aspectos centrales de la epistemología de este filósofo de la ciencia italiano.

Sin hacer aportaciones totalmente originales sobre el concepto de objetividad científica, Agazzi, constata que la mayoría de las interpretaciones científicas actuales, favorecen una visión objetivista de la ciencia (Agazzi, 2014: 57). Por objetividad científica, el filósofo italiano entiende al objeto científico que, pudiendo ser abstracto y formal, según los protocolos específicos de cada ámbito científico, siempre necesita una referencialidad empírica que garantice su carácter real en el espacio y en el tiempo físico (Sanguineti, 2015: 2-3). En otras palabras, la objetivación sería el producto de la actividad humana del sujeto que se acerca espontáneamente al conocimiento y busca un referente concreto e inmediato a las afirmaciones lingüísticas expresadas; todo esto con la mediación de la intrínseca capacidad humana de abstraer y teorizar.

Sin embargo, como lo afirma Juan José Sanguineti (2015), esto no significa que en el quehacer científico no puedan existir también «objetos sólo intencionales, sin una referencia al mundo físico» (3) y con la posibilidad de un conocimiento objetivo ya que, para Agazzi, no hay parte de la realidad toda que no exija una consideración desde la objetivación (Agazzi, 2014: 282). Este tipo de objetos intencionales, que forman parte de las diferentes disciplinas, no entienden la verdad como la clásica correspondencia entre lo pensado y la cosa concreta. Es la correspondencia o adecuación entre lo pensado y la cosa tangible que el autor italiano denomina objetividad en sentido fuerte. Según esta manera de entender la objetividad, una propiedad material se encuentra realmente en el objeto que se investiga. En consonancia, tendremos que considerar objetivo el discurso que es capaz de describir dichas características del objeto (Agazzi, 2015: 54). Este significado de objetividad es el que se ha impuesto, en el sentido que se les otorga a las ciencias experimentales modernas, en cuanto a sus posibilidades, procedimientos metodológicos y alcances praxeológicos. La objetividad en sentido fuerte es una característica propia y necesaria de las ciencias experimentales; pues la capacidad de explicar y describir los objetos que indagan pertenece a la naturaleza epistemológica de las ciencias, que tiene a la experiencia como su fundamento. Sin embargo, esta perfectiva epistemológica 
no agota, en modo alguno, a la realidad; tampoco constituye la única forma de acercarse a una realidad caracterizada por su intrínseca complejidad.

Por eso, además del sentido fuerte de objetividad científica, Agazzi distingue un sentido débil, cuando «la objetividad es la propiedad de un discurso cuya validez no depende del sujeto (...) sino de una cierta totalidad de sujetos. En otras palabras, corresponde a la intersubjetividad sin referencia a objetos» (Agazzi, 2014: 64). Es en este sentido que el autor observa que hay una ciencia contemporánea que busca una objetividad sin objetos materiales. Es notorio que este segundo significado de objetividad deja espacio para los esfuerzos investigativos provenientes de aquellas ciencias cuya naturaleza epistemológica no contempla la manipulación de objetos empíricos, sino que debe tratar con entes de razón ${ }^{1}$ cuya existencia material y tangible es irrelevante cuando se trata de medir los niveles de cientificidad y objetividad que pueden alcanzar.

\subsection{Hacia una objetividad abierta: aportes desde la hermenéutica}

Ante la, así llamada, crisis en los fundamentos de las ciencias exactas y experimentales de finales del siglo XIX e inicios del siglo XX, muchos hombres de ciencia y epistemólogos consideraron que la objetividad absoluta en sentido fuerte, anteriormente caracterizada, era una finalidad muy difícil de alcanzar en su plenitud. Un ejemplo paradigmático de esta postura es la de Karl Popper, quien exige que las hipótesis deben ser falsables, es decir si la clase de sus falsadores no es una clase vacía, se exige que los enunciados tengan la propiedad en potencia de ser falsables; los enunciados que no tienen la propiedad de ser falsables no pertenecen a la ciencia y por tanto no son objetivos, así los enunciados que son lógicamente imposibles de falsar no pertenecen al campo científico (Mejía Soto, 2004).

\footnotetext{
1 Como afirma Óscar Barroso Fernández (2011), aquello a lo que se refieren los entes de razón constituyen «elementos fundamentales para el conocimiento... (ya que) el ente de razón se identificará con el imposible lógico y no con el imposible existencial científico» (p. 141). Es decir que, sobre los entes de razón es perfectamente posible y hasta necesario investigar desde el punto de vista científico, pues ya se hace uso de ellos en el quehacer cotidiano de la labor académica, científica e investigativa. Desde la concepción de Suárez, entes de razón pueden ser algunos principios matemáticos, las negaciones y las privaciones (Suárez, 2011: 142).
} 
Ante esta postura el reto para los epistemólogos fue la de fundamentar mejor la objetividad en su sentido débil (Agazzi, 2015: 54), que es propia de las ciencias humanísticas. Dicho esfuerzo inicia con los aportes hermenéuticos de Wilhelm Dilthey y su intento de dar personería epistémica a las ciencias del espiritu cuando, desde el pensador alemán, se sostiene que «aunque todas las ciencias son ciencias empíricas (...), la experiencia encuentra su coherencia y su validez en el apriori estructurante de nuestra conciencia» (Grondin, 2002: 129). Es por este motivo que las condiciones objetivas de validez de las ciencias humanas se cimientan en la experiencia interior. A esta experiencia interior de Dilthey hoy se la puede llamar intuición o corazonada cientifica y constituye el inicio y detonante de todo proceso científico. En la misma línea de búsqueda del estatuto epistemológico para las ciencias humanas, Hans-Georg Gadamer, inicia afirmando que cada ciencia debe buscar sus propios métodos para gozar del estatus de ciencia; sin embargo, el hermeneuta alemán, habla de que el proceder metodológico que caracteriza a las ciencias del espíritu, tiene que ver con un tacto procedimental que no se somete a ninguno de los métodos de investigación científica conocidos. De aquí que, la obra de Gadamer titulada Verdad y Método, constituye una crítica a la obsesión metodológica para justificar la cientificidad de las ciencias humanísticas (Grondin, 2002: 160). El tacto procedimental que deben tener las ciencias humanas no puede dejar de recordar el proceso de formación histórica de las diversas formas de entender y practicar la ciencia. En dicho proceso de conformación, por la influencia de la estética kantiana, se habría dado una subjetivación y estetización de los conceptos más importantes del humanismo, lo que habría desembocado en la pérdida de objetividad y desacreditación del conocimiento proveniente de las ciencias humanas. Adicionalmente, esto hizo que se profundice la separación epistémica de las ciencias humanas (cuyo ámbito de indagación rayaba en lo meramente subjetivo y estético) y las ciencias naturalesexperimentales que se consideren como el prototipo de la objetividad y seriedad científica (Grondin, 2002: 160-161). Para Gadamer, una adecuada concepción de las ciencias humanas y su pertinente configuración científica, será el resultado de una erradicación de la exagerada estetización y subjetivación en la concepción y en la práctica científica contemporánea.

La intervención del sujeto que está constantemente interpretando es una de las características de la ciencia contemporánea, tanto de las ciencias naturales como de las ciencias humanas. De hecho, para Agazzi lo que caracteriza a las ciencias humanas (o a todo género de ciencia), es la identidad entre sujeto y objeto, ya que es el sujeto quien es capaz de alterar los datos «debido a la implicación emocional y a las expectativas previas a la recogida del dato; la existencia de la 
libertad; la existencia de fines; la dimensión hermenéutica, o sea, la necesidad de interpretar los fenómenos humanos» (Artigas: 2002: 191). Adicionalmente, Agazzi sostiene que los mismos criterios científicos fundamentales se aplican tanto en las ciencias naturales como en las humanas. Así, por ejemplo, también en las ciencias humanas se pueden establecer criterios operacionales; se pueden utilizar modelos en los que se prevean desviaciones de modo que se consigan estadísticas válidas; también en las ciencias experimentales, como en las humanidades, es necesario interpretar y realizar experimentos. En definitiva, es posible una complementación y un diálogo epistemológico y hermenéutico entre las ciencias naturales-experimentales y las ciencias humanas (Artigas: 2002: 191).

Sin embargo, eso no significa que hay que equiparar los dos tipos de ciencia. Pues, en la medida en que se estudien los fenómenos típicamente humanos en los que intervienen características tales como la autoconciencia y la libertad, será necesario alejarse del método experimental que se emplea al estudiar las pautas espacio-temporales materiales.

En la práctica científica y académica de nuestros días se puede constatar que el sentido fuerte de la objetividad científica, descrito por Agazzi, continúa vigente ya que, como escribe el autor italiano, "todos admitimos que cada ciencia se ocupa, no de la realidad en cuanto tal, sino sólo de sus objetos específicos» (Agazzi, 2015: 55). El pensador italiano afirma que, es la dedicación a lo particular de cada ciencia y los acuerdos estandarizados logrados en cada ámbito específico del conocimiento, lo que nos permite establecer criterios de verdad y criterios de referencia (Agazzi, 2015: 55). Por eso, es de relevar el hecho de que contribuye con sus ideas en la construcción de un concepto de objetividad abierto y contextualizado. En consecuencia, no sería científicamente objetivo aplicar criterios de verdad o de referencia en manera indiferenciada a disciplinas cuya naturaleza epistémica es esencialmente distinta a la de las ciencias experimentales. Para ilustrar esto, Agazzi presenta el caso de la mecánica clásica y la mecánica cuántica cuando «utilizan criterios operacionales muy distintos para determinar el valor de sus magnitudes y, por tanto, se refieren a objetos distintos, aunque los nombren con la misma palabra» (Agazzi, 2015: 55). La necesidad de diferenciar los contextos en los que se realiza la investigación es un requisito importante para alcanzar una objetividad científica. Por consiguiente, para Agazzi,

las dos formas de objetividad, fuerte y débil, coinciden (...) y una vez entendido que la verdad de un discurso siempre es relativa a los referentes del mismo y no a una supuesta realidad general, puede recuperarse plenamente 
la noción de verdad en la ciencia. La verdad no resulta eliminada en favor de la objetividad, sino que a través de este concepto puede ser precisada y definida (Agazzi, 2015: 55).

La verdad objetiva, en ciencia, es un horizonte que puede alcanzarse en las diversas formas de indagación sobre lo real y gracias a la peculiaridad de la inteligencia humana de diferenciar y valorar cada ámbito del saber en su campo específico y con análoga posibilidad de alcanzar certezas objetivas.

Es de aquí que son epistemológicamente disonantes aquellas voces que juzgan y discriminan determinados ámbitos del conocimiento -las humanidades, por ejemplo-, debido a que no siguen los mismos estándares metodológicos de aquellos otros ámbitos del saber cuya naturaleza es diferente, como es el caso de las ciencias experimentales.

\subsection{La objetividad y el estatuto científico de las ciencias humanas}

La concepción epistemológica de Evandro Agazzi contiene una constante preocupación por clarificar el estatuto científico de las ciencias humanas en el contexto académico y científico actual.

En el caso de una disciplina de naturaleza abstracta, con proposiciones enteramente teóricas, se debe discernir la factibilidad epistemológica de que, a partir de dichas proposiciones teóricas y de acuerdo con la naturaleza objeto indagado, exista la posibilidad de tomar un camino lógicamente correcto que incluya procesos de comprobación, no solo argumentativa, sino también empírica. Un ejemplo de este tipo de ciencias, propuesto por Agazzi, es la historia: en esta disciplina no todas las proposiciones son de naturaleza medible, unívoca, física u observable, pues, los contenidos de varios documentos deben verificarse a través de una compatibilidad lógica con los hechos documentados y, posiblemente, pueden ser refutadas por el descubrimiento de nuevos documentos. Si las reconstrucciones históricas carecen de esta posibilidad, deben calificarse como no-históricas y, por lo tanto, ficticias (Agazzi, 2015: 13). También los valores humanos entran en la misma dinámica epistemológica y argumentativa. En efecto, la reflexión axiológica, que es estructuralmente humana, hace que el ser humano se pregunte por lo valioso y lo que no tiene valor; lo malo y lo bueno; lo positivo y lo negativo; 
lo mejor y lo peor. Para responder a estas preguntas, los humanos han elaborado diversos sistemas teóricos de razonamiento e interpretación (doctrinas éticas, sistemas legales, teorías económicas, etc.). Ante esta realidad, Agazzi afirma que

Es perfectamente legítimo, e incluso altamente deseable que, dentro de las regiones ontológicas caracterizadas por tales atributos no empíricos, se promueva una confrontación racional precisa y objetiva, recurriendo a las herramientas intelectuales de la intuición y el uso sintético de la razón que se pueden aplicar fructíferamente también fuera de la ciencia (Agazzi, 2015: 13).

Esa confrontación epistémica, necesita aplicar las condiciones generales del conocimiento humano como: el pluralismo epistemológico y ontológico, la aceptación de las ontologías regionales, el uso sintético de la razón y la mediación de la experiencia. Con estas bases epistemológicas se puede vislumbrar la constitución de una ciencia más dialógica, complementaria, integral e inclusiva, que no excluya racionalidades no convencionales para que contribuya a un progreso científico más sostenible y menos invasivo con la naturaleza y su necesario equilibrio.

Las condiciones generales del conocimiento tienen una constitución ontológica y no son propiedad exclusiva de ninguna ciencia particular. Están, en germen, en la experiencia sensorial y son captados por la intuición intelectiva. Por medio de la intuición intelectual, se pueden formular ciertos principios generales, los cuales se presentan como la base de la generalidad y que, por lo tanto, configuran lo que Agazzi denomina ontología general (Agazzi, 2015: 13). Es a partir de aquí que Evandro Agazzi, en la línea de Aristóteles y de Kant, propone las fases para la producción del conocimiento científico de los diversos tipos de ciencia y que se funda en una racionalidad ensanchada en sus horizontes. Dichos pasos no son necesariamente cronológicos, ni consecutivos. El primer paso sería el partir de la experiencia sensorial; el segundo la aplicación consistente de los principios generales; el tercer la aplicación del uso sintético de la razón y el cuarto es el reconocimiento de que hay entidades que no comparten los atributos de los objetos empiricos y, por lo tanto, pertenecen a la totalidad de lo que realmente existe (Agazzi, 2015: 14).

Agazzi, demostrando su admiración y la validez de la tradición epistemológica y metafísica clásicas, afirma que 
este es el camino que han intentado varias tendencias de la metafísica tradicional, cuyo programa es metodológicamente inobjetable. Probablemente no serán inmunes a los puntos débiles y no obtendrán la certeza absoluta de que, a menudo, fingieron haber conquistado, pero en nuestros días somos conscientes de que las ciencias están lejos de haber alcanzado un grado tan alto de certeza. Por lo tanto, es mucho más razonable aceptar que la razón se use bajo sus diversas formas, con la conciencia de que los humanos están sujetos al error, expuestos a dudas e ignorancia en cada dominio (Agazzi, 2015: 14).

La actitud innata de búsqueda permanente es lo que originó el nacimiento y el desarrollo de la ciencia occidental. Esa misma actitud posibilitó el gran desarrollo de las ciencias, tanto experimentales como humanas. El camino científico que la humanidad ha recorrido durante más de dos milenios ha ofrecido resultados que están entre lo satisfactorio, lo funcional, lo alienante, lo deshumanizante, lo invasivo, lo paradójico. Debido a esto, urge la necesidad de explorar nuevos rumbos, de aventurarse en nuevas empresas epistémicas, con la consciencia de que el error y la equivocación son parte del camino y que la verdad que se aspira alcanzar será siempre parcial e incompleta; constituyéndose un punto de partida y nunca punto de llegada definitivo e incambiable. En el camino, el punto provisional alcanzado, es la convicción de que las racionalidades «no deben oponerse entre sí, sino más bien cooperar con la visión de una mejor comprensión de nuestro mundo y una mejor orientación de nuestras vidas» (Agazzi, 2015: 14).

\section{Objetividad e intersubjetividad científica}

Evandro Agazzi constata que, el sentido más extendido de objetividad, tanto en la ciencia como en la sociedad contemporánea, es el que lo identifica con el clásico concepto de intersubjetividad cientifica ${ }^{2}$. Es decir, la consideración del

\footnotetext{
2 Sobre la identidad entre intersubjetividad y objetividad, Karl Popper es bastante elocuente en su famosa obra Die beiden Grundprobleme der Erkenntnistheorie [Los dos problemas básicos de la epistemología], pero también en la obra La sociedad abierta y sus enemigos, donde afirma que «la objetividad se halla íntimamente ligada al aspecto social del método científico, al hecho de que la ciencia y la objetividad científica no resultan (ni pueden resultar) de los esfuerzos de
} 
quehacer científico, tanto en sus procesos, en sus métodos y en sus resultados, como un discurso público que debería facilitar la transmisión de información científica, con la posibilidad de cooperación internacional a través de los intercambios entre especialistas en ámbitos relacionados con experimentos, teorías e incluso resultados científicos. Es esta relación científica intersubjetiva y abierta lo que otorgaría objetividad a las afirmaciones científicas (Agazzi, 2014: 57-58). Según este criterio, la dimensión intersubjetiva de un conocimiento o un procedimiento científico, debe ser el filtro fundamental para evaluar la solidez, el impacto y la validez de los aportes científicos, tanto en los procedimientos metodológicos como en los resultados y consecuencias prácticas.

El quehacer científico no es un hecho privatizable, ni de parte de sujetos, ni por parte de instituciones corporativas. Toda la ciencia que se construye a nivel de sujetos o a nivel de entidades corporativas tiene una dimensión social y abierta; esto no sólo en los procesos internos y técnicos del quehacer científico, sino también en sus resultados y consecuencias prácticas. De aquí que no caben aquellas investigaciones científicas cargadas de secretismos; pues, tarde o temprano los efectos de tales procesos o de los resultados investigativos terminarán afectando a la sociedad. Por eso, en el espíritu de Karl Popper, se puede afirmar que todo el quehacer científico es conocimiento de carácter público y abierto a ulteriores críticas, observaciones y complementaciones de forma o de fondo. Dichas complementaciones pueden provenir del mundo de la ciencia o de la academia especializada, pero también pueden provenir de las ciencias que, sin ser especializadas, pueden dar su aporte ya sea sobre el contenido o sobre aspectos formales acerca del objeto de estudio del que se trate.

Mariano Artigas, afirma que la intersubjetividad tiene que ver con el hecho de que

es preciso relacionar los conceptos básicos de una teoría con los experimentos que sirven para definirlos y para medir sus valores. Cuando establecemos esas relaciones, acotamos un ámbito de intersubjetividad, porque cualquier persona puede utilizar los conceptos así definidos en el mismo

un hombre de ciencia individual por ser objetivo, sino de la cooperación de muchos hombres de ciencia. Puede definirse la objetividad científica como la intersubjetividad del método científico» (Popper, 1982: 430). 
sentido que nosotros lo hacemos y llegando a los mismos resultados. El acuerdo sobre el uso de los conceptos conduce a la intersubjetividad, y en la ciencia experimental se exige establecer ese tipo de acuerdos que sirven como base a la peculiar objetividad de la ciencia experimental (Artigas, 2009: 224).

Lo anterior, no significa que sólo sea real y objetivo lo que se puede indagar siguiendo con rigor el método de las ciencias experimentales. Pues, desde Karl Popper (1982), si el quehacer científico no sale de lo individual para asumir, conscientemente, el carácter social lo que se llama «objetividad científica debe convertirse (...) en algo completamente incomprensible si no imposible, y no sólo en las ciencias sociales o políticas, donde pueden desempeñar algún papel los intereses de clase y otros móviles ocultos semejantes, sino también en las ciencias naturales» (430).

En definitiva, al salir de los estrechos horizontes del investigador individual y el tener la capacidad de confrontarse con los demás, implica que todo quehacer científico debe ser repetible por parte de cualquier investigador. Este carácter público de la ciencia implica que, toda ella, está sujeta a la discusión y la crítica intersubjetiva. A esto hay que acostumbrarse en el mundo de la academia y en el de la investigación científica; para ello, el profesional investigador o académico debe ser capaz de superar, con madurez humana y epistémica, el ámbito personal del profesional-científico.

No cabe duda que la definición de los objetos de investigación es esencial en el quehacer científico. Pero igual importancia tiene la conciencia de que, no todos los objetos de indagación son iguales o pertenecen al mismo ámbito epistémico. Algunos ámbitos de las ciencias experimentales gozan de una especial objetividad (Artigas, 2009: 224). Esta objetividad se deriva de la rigurosidad en el control experimental, de la relacionalidad y apertura de los procesos experimentales y del acuerdo-consenso que esto produce en la comunidad científica, investigativa y social, a nivel local e internacional.

Finalmente, cabe señalar que, sin caer en el escepticismo o en el pesimismo epistémico, sino más bien asumiendo una postura realista y objetiva sobre las reales posibilidades de la mente humana en cuanto al conocimiento, Popper dice que el «antiguo ideal científico de la epistémè-de un conocimiento absolutamente seguro y demostrable- ha mostrado ser un ídolo. La petición de objetividad 
científica hace inevitable que todo enunciado científico sea provisional para siempre» (Popper, 1980: 261). Es de este carácter intersubjetivo y provisional del conocimiento científico de donde se deriva la perenne novedad y el progreso científico, desde la perspectiva de Popper y de Evandro Agazzi.

\section{Objetividad como universalidad y necesidad ontológica}

La objetividad como intersubjetividad se ubica en las concepciones epistemológicas según las cuales no hay lugar para las certezas absolutas, ya que todo el conocimiento es dinámico, revisable y perfectible. En este contexto con visos de cierto relativismo epistémico, Agazzi se pregunta si aún sería posible hablar de objetividad en un sentido más ontológico, más esencial, con un carácter de universalidad y necesidad.

Desde la física clásica, el hecho de que exista la característica de la invariancia ${ }^{3}$ defendida, entre otros, por Galileo, Newton y Max Born, como una auténtica marca de objetividad, recuerda las viejas exigencias ontológicas de la permanencia, universalidad y necesidad (Agazzi, 2014: 60). Esto implicaría que, algunas leyes científicas son concebidas como universalmente válidas y permanentes dentro de su campo específico de aplicación. Todo esto, con la finalidad de subrayar que es aceptable la descripción fenoménica de los objetos investigados, pero es muy importante no olvidar los diferentes marcos de referencia que se adoptan.

No obstante, según el parecer de Evandro Agazzi, «resulta que estas diferentes proyecciones del mismo objeto pueden ser sometidas a ciertas reglas de transformación que constituyen grupos, en el sentido matemático de esta palabra» (Agazzi, 2014: 61). Tal sentido matemático es entendido como la existencia de categorías o principios abstractos que conservan la huella de la universalidad y la permanencia necesaria. Desde la ontología tradicional se puede hablar de «permanencia básica de la estructura de la realidad» (Agazzi, 2014: 61). Por lo tanto, como parte de la constitución ontológica de la realidad total, junto con la permanencia, abría algo que no se somete a la ley de la mutación y el cambio continuo.

3 Las leyes básicas de la física tienden a mantenerse más allá del tiempo y del espacio ya que son las mismas en todos los sistemas de referencia inerciales. 
Este tipo de objetividad ontológica, es denominada por Agazzi objetividad en sentido débil, ya que subrayaría las características de la universalidad y la necesidad, que serían las que menos aluden a la referencia a los objetos (Agazzi, 2014: 61) $)^{4}$. Claro está que el autor es consciente de que la objetividad en sentido fuerte (Agazzi, 2014: 62), que recuerda al concepto clásico de verdad, entendida como la adecuación entre la idea y la cosa concreta. Este segundo sentido de la objetividad es pertinente para las ciencias naturales y experimentales, mientras que la objetividad ontológica tendría la finalidad de expresar, primordialmente, el lado epistemológico de la objetividad. Los significados de la objetividad son utilizados y buscados naturalmente en los procesos de investigación científica, sin que exista contradicción y supremacía de un significado sobre otro.

Para nadie es un secreto que el actual quehacer científico, académico e investigativo, privilegia el sentido fuerte de la objetividad, presuponiendo (y a veces olvidando) la existencia del significado ontológico de la objetividad científica (Agazzi, 2014: 62). Es a partir de este hecho que es importante formar epistemológicamente a los investigadores y académicos contemporáneos para que el quehacer científico, en su compleja estructura ejecutiva, sea consciente de las bases teóricas y técnicas que proporciona la objetividad en si significado débil. Con esto, se evitarían los reduccionismos epistemológicos contemporáneos que exigen a la ciencia, en su procesos y resultados, la estricta correspondencia entre las ideas formales y las cosas materiales, cayendo en utilitarismos que rayan en lo superficial y efímero, exponiendo al ser humano a un tempranero encuentro con el sin sentido y el tedio por la vida.

Por otro lado, y como consecuencia de las visiones reduccionistas de la ciencia y de la realidad, a los saberes que no incluyen la estricta correspondencia o referencialidad entre las ideas formales (teorías, hipótesis, enunciados científicos, etc.) y la cosa concreta (resultados, artefactos tecno-científicos, etc.) son saberes que la ciencia contemporánea los minusvalora y desecha por considerarlos carentes de objetividad.

4 Esto no significa que el autor no postule la posibilidad de proponer una ontología del objeto científico, al contrario, se habla de la necesidad de elaborar una ontología con estas características y presenta sintéticamente sus contribuciones para esta tarea: «tuve que aprovechar de una verdadera teoría referencial de la verdad. Además, tuve que considerar también un segundo sentido distinto de objeto científico, según el cual es una entidad abstracta obtenida a través de una idealización, de la que los únicos referentes concretos son ejemplificaciones...» (Dilworth and Agazzi, 2014: 333). 
En cuanto al tema de la objetividad, en las dos significaciones antes aludidas, lo pertinente en evitar los extremos que rayan entre el realismo ingenuo (exigencia de adecuación perfecta entre los insumos formales de la ciencia y la realidad concreta) y lo triunfalista que presupone una concepción demasiadamente simplista de la realidad.

\section{Objetividad, realismo científico y pluralismo epistemológico}

De acuerdo al realismo científico de Evandro Agazzi, todas las ideas, los conceptos, las intuiciones, definiciones y demás entes de razón, que preceden y acompañan al quehacer científico, no son sólo ideas abstractas, sin una existencia real. Con esto, el autor se hace eco de una visión epistemológica optimista del quehacer científico, en cuanto estimula la búsqueda de los científicos e investigadores. Sin embargo, aunque la objetividad en sentido fuerte coincide con aquello que es empíricamente captable a través de los sentidos externos, sin embargo, también tienen el estatuto de reales «aquellos entes teóricos no inmediatamente observables que vienen normalmente introducidos por las ciencias para explicar los fenómenos observables» (Agazzi, 2009: 45). Es decir, también estos entes teóricos tienen cierto grado de objetividad, pues se los utiliza en el quehacer científico como insumos y puntos de partida básicos que cimientan el trabajo científico y académico. La consistencia existencial de los entes teóricos, así como su pertinencia epistémica, ha sido negada o declarada como innecesaria por parte de ciertas prácticas académicas y pedagógicas contemporáneas con claros rezagos del empirismo, utilitarismo y pragmatismo radical. Esto último alude al problema epistemológico, aún poco desarrollado, de la relación entre la teoría y la praxis. Es notorio que en nuestros días asistimos a una cierta discriminación hacia la teoría y a un ensalzamiento de todo lo que es práctica, resultados e implementos útiles. En este orden de ideas, «toda buena praxis proviene de una buena teoría y toda buena teoría desemboca en una praxis» (Bolaños, 2015: 34).

En definitiva, el realismo científico de Evandro Agazzi implica el reconocimiento de que la realidad científica a indagarse, debido a su intrínseca complejidad, está compuesta por varios niveles y que, por eso, no caben reduccionismos del nivel ontológico-real al nivel empírico-observacional. Más bien, en el quehacer tecno-científico contemporáneo, es imprescindible y necesaria la recíproca colaboración entre los diferentes niveles de la realidad, la 
cual debe ser entendida en manera integral e interdisciplinaria. De esta manera, afirma Agazzi:

...el realismo defendido por mí implica el reconocimiento de la multiplicidad de los niveles de realidad, la irreductibilidad ontológica de los unos a los otros, incluso en la conciencia de sus recíprocos espacios, en el contexto de un horizonte complejo de la realidad asumida en manera global (Agazzi, 2009: 47$)^{5}$.

Es a partir de esta concepción realista y abierta de Evandro Agazzi, que es admisible y necesario el pluralismo epistemológico. Esta concepción se basa en el reconocimiento de una gran variedad de criterios de objetivación que se constituyen en juicios de referencialidad y de verdad para tal o cual ámbito epistemológico específico (Agazzi, 2009: 46). La diversidad en los criterios de objetivación implica que los científicos, investigadores y académicos amplíen sus horizontes epistemológicos, considerando de manera igualitaria los diferentes saberes y concepciones del mundo, tanto los científicos como los no científicos, en el sentido occidental de la expresión. Caso contrario, la exclusión de ciertos saberes y concepciones del mundo en el ámbito de las discusiones y decisiones socio-políticas representaría una injusticia epistémica que, como señala Boaventura de Sousa Santos, está en la base de toda injusticia social (Velasco, 2016: 70-71). He aquí el significativo texto de De Sousa Santos:

Ya que el conocimiento científico no está socialmente distribuido de manera proporcionada, las intervenciones en el mundo real que favorece tienden a ser aquellas que atienden a los grupos sociales que tienen acceso al conocimiento científico. La injusticia social se basa en la injusticia cognitiva.

\footnotetext{
5 He aquí el texto original que contiene una intraducible claridad: «...il realismo da me difeso implichi il riconoscimento della molteplicità dei 'livelli di realtà, l'irriducibilità ontológica degli uni agli altri pur nella consapevolezza dei loro reciproci intrecci nel contesto di un orizzonte complesso della realtà globalmente presa» (Agazzi, 2009: 47). La traducción es de Robert Fernando Bolańos Vivas.
} 
Sin embargo, la lucha por la justicia cognitiva no tendrá éxito si se sustenta únicamente en la idea de una distribución más equilibrada del conocimiento científico... [se requiere] reconocer formas alternativas de conocimiento y de interconectarse con ellas en términos de igualdad (51).

Es evidente que la construcción del objeto de investigación es una tarea multidimensional ya que, este hecho tiene consecuencias en la vida social, política y económica de las naciones. El pluralismo epistemológico, presupone y necesita un trabajo inter y multidisciplinario que sea capaz de hacer efectivo el ensanchamiento de la razón. Así lo escribe Fabio Minazzi al referirse a la epistemología agazziana:

el objeto científico no puede dejar de emerger como una construcción intelectual peculiar. Pero el reconocimiento mismo de la existencia de este constructo intelectual no sólo le ha llevado a aclarar la naturaleza eminentemente relacional de la verdad, sino también las razones de un realismo crítico que no puede sino aceptar una perspectiva orientada a salvaguardar un pluralismo epistemológico válido, capaz de identificar la multiplicidad de diferentes niveles de realidad investigados y estudiados por diferentes disciplinas científicas (Minazzi, 2015: 13).

El pluralismo epistemológico defendido por Evandro Agazzi, no implica una aceptación acrítica o relativista de la realidad científica que contiene una infinidad de rasgos perceptivos y una variedad ilimitada de formas predicativas. Sostener esto, podría desembocar en un relativismo epistemológico ya que, no sería otra cosa que una insostenible expresión del dualismo epistemológico (Agazzi, 2014: 217). Comprender el espíritu del pluralismo epistemológico y de la opción agazziana por el realismo científico, implica «el reconocimiento de la pluralidad de criterios de objetivación, que son al mismo tiempo criterios de referencialidad y de verdad» (Agazzi, 2009: 46). Los significados de lo verdadero, lo científico y lo real en la ciencia contemporánea, no pueden privilegiar un significado unidireccional y cerrado, sino que es necesario tener una mentalidad cosmopolita y abierta a la complementariedad de perspectivas. 
Es por eso que, para explicar el sentido epistemológico de lo real, es pertinente el viejo recurso a la analogía al que recurre Agazzi, parafraseando de la siguiente forma a Aristóteles: «si ciencia se dice de muchas maneras, también real se dice de muchas formas» (Agazzi, 2009: 46). Para una producción e investigación científica pertinente, no cabe permanecer en una concepción epistemológica cerrada al concebir a lo científico sólo como aquello que se somete rigurosamente a la verificación y al control experimental, desechando otras formas de racionalidad y de rigurosidad científica.

\section{Conclusiones}

El análisis del concepto de objetividad científica permite aportar elementos para que su configuración sea más abierta e incluya y justifique el quehacer de las diferentes ciencias. Si bien es cierto que es admisible la distinción de una objetividad en sentido fuerte (el control empírico experimental), y otra en sentido débil (las bases ontológicas del conocimiento), estas distintas formas de entenderla no constituyen un problema si se tiene en cuenta la especificidad epistemológica de los ámbitos contextuales en los que se desarrolla la actividad científica. Es a partir de esta concepción de ella, abierta a la complementariedad epistémica, que puede provenir de ámbitos que no siguen los criterios clásicos de cientificidad occidentales, que es posible ir construyendo un quehacer científico más inclusivo e integral y por eso, más pertinente a los múltiples desafíos de la realidad actual.

Se evitaría la consideración discriminante hacia las ciencias, cuya naturaleza no se sustenta en la cuantificación de datos experimentales, si los operadores de la ciencia estuvieren dotados de una formación epistemológica que les permita comprender y buscar objetividad de acuerdo a la naturaleza de la ciencia en la que operan. De aquí que es pertinente un nuevo interrogante: ¿Qué está pasando con la formación epistemológica de los operadores de ciencia y tecnología, a tal punto de no poseer las herramientas conceptuales suficientes como para hacer una aplicación de las construcciones científicas en manera contextualizada? Es urgente que los operadores de ciencia y tecnología reciban una formación epistemológica que les permita estar abiertos a las complementaciones epistemológicas de las diferentes ciencias.

No cabe duda de que el quehacer científico que privilegia la objetividad científica, en sentido fuerte, identificándola con la comprobación experimental 
y la producción de resultados prácticos y útiles, contiene muchos elementos que desafían a la razón. En efecto, es evidente que la ciencia que sólo se identifica con lo empíricamente contrastable y lo utilitariamente rentable, deja afuera de su espectro una serie de situaciones que también son parte de la vida humana (creencias, valores, utopías, ocio, etc.). Esto significa que estos aspectos de la vida humana que, al no ser cientificos, ¿son totalmente irracionales? En consecuencia, se abren nuevos campos de indagación epistemológica como aquel de la relación entre racionalidad y tecnociencia o aquel de la necesidad de ampliar la concepción de racionalidad humana ante la existencia e irrupción de otras formas alternativas de racionalidad.

\section{Bibliografía}

AgAZZI, Evandro (2009). "Che cosa è dentro e che cosa è fuori dalla scienza. Una riflessione filosófica”. Annuario filosofico, no 25, pp. 27-63.

-(2014). Scientific Objectivity and Its Contexts, New York: Springer. Consultado el 27 de febrero de 2018, D: 10.1007/978-3-319-04660-0.

-(2015). “En busca de la objetividad." Investigación y ciencia, n 466, pp. 51-65.

-(2015). Rationality outside science, correo electrónico recibido por el autor: 06, marzo, 2015.

Agazzi, Evandro y Dilworth, Craig (2014). "Objectivity, truth and scientific realism”. Epistemologia, rivista italiana di filosofia della scienza, XXXVII, pp. 325-336, D:10.3280/EPIS2014-002010.

AlaI, Mario (2006). "Il realismo scientifico di Evandro Agazzi”. Isonomia, pp. 77-90.

Artigas, Mariano (2002). Filosofía de la ciencia. Navarra: Eunsa.

Barroso, Óscar (2011). "Los entes de razón en Suárez. Una concepción barroca de la realidad". Anales del Seminario de Historia de la Filosofía. Vol. 28, pp. 135-161.

BolaÑos, Robert (2015). "Elementos de hermenéutica y fenomenología para un diálogo metodológico entre las ciencias". Sophia: Colección de Filosofía de la Educación, $\mathrm{n}^{\circ} 19$, pp. 25-46.

Bolaños, Bernardo (2002). Argumentación científica y objetividad. México: Editorial Doctor Jiménez.

De Sousa Santos, Boaventura (2010). Refundación del Estado en América Latina. Perspectivas desde una epistemología del sur. Bogotá: Siglo XXI.

Grondin, Jean (2002). Introducción a la hermenéutica filosófica. Barcelona: Herder. 
Mejía Soto, Eutimio (2004). "Aproximación al concepto de objetividad en Karl Popper". Recuperado de https://www.gestiopolis.com/aproximacion-al-conceptode-objetividad-en-karl-popper/

Minazzi, Fabio (2015). "Evandro Agazzi Philosopher. An Overview of His Thought". Science Between Truth and Ethical Responsibility, Evandro Agazzi in the Contemporary Scientific and Philosophical Debate, ed. Mario Alai, Marco Buzzoni, and Gino Tarozzi. New York: Springer. Doi: 10.1007/978-3-319-16369-7.

Popper, Karl (1980). Lógica de la investigación científica. Madrid: Tecnos.

—(1982). La sociedad abierta y sus enemigos. Barcelona: Paidós.

Sanguineti, Juan (2015). "Scientific Objectivity and Its Contexts", reseña de Scientific Objectivity and Its Contexts. Anuario Filosofico, 48.1, pp. 2-3.

Velasco, Ambrosio (2016). "Diversidad cultural, pluralismo epistémico, ciencia y democracia. Una revisión desde la filosofía política de las ciencias”. Acta sociológica, $n^{\circ} 71$, pp. 51-78.

Recibido: 27/09/2018

Aceptado: 18/10/2019

\section{(c) $(1) \Theta$}

Este trabajo se encuentra bajo una licencia de Creative Commons Reconocimiento-No Comercial-SinObraDerivada 4.0 